\title{
Trends in and disparities for acute myocardial infarction: an analysis of Medicare claims data from 1992 to 2010
}

\author{
Jasvinder A Singh ${ }^{1 *}$, Xin Lu², Said Ibrahim ${ }^{3}$ and Peter Cram ${ }^{4,5}$
}

\begin{abstract}
Background: It is unknown whether previously reported disparities for acute myocardial infarction (AMI) by race and sex have declined over time.

Methods: We used Medicare Part A administrative data files for 1992 to 2010 to evaluate changes in per-capita hospitalization rates for AMI, rates of revascularization (percutaneous coronary intervention (PCI) and coronary artery bypass grafting (CABG)), and 30-day mortality for four distinct patient subcohorts: black women; black men; white women; and white men, adjusted for age, comorbidities and year using logistic regression.

Results: The study sample consisted of 4,045,267 AMI admissions between the years 1992 and 2010 (166,660 black women; 116,201 black men; 1,870,816 white women; 1,891,590 white men). AMl hospitalization rates differed significantly in 1992 to 1993 among black women (61.6 hospitalizations per 10,000 Medicare enrollees), black men (73.2 hospitalizations), white women (72.0 hospitalizations) and white men (113.2 hospitalizations) $(P<0.0001)$. By 2009 to 2010 AMI hospitalization rates had declined substantially in all cohorts but disparities remained with significantly lower hospitalization rates among women and blacks compared to men and whites, respectively ( $P$ <0.0001). In multivariable-adjusted analyses, despite narrowing of the differences between cohorts over time, disparities in AMI hospitalization rates by race and sex remained statistically significant in 2009 to $2010(P<0.001)$. In 1992 to 1993 and 2009 to 2010, rates of PCI within 30-days of AMI differed significantly among black women (8.6\% in 1992 to $1993 ; 24.2 \%$ in 2009 to 2010), black men (10.4\% and 32.6\%), white women (12.8\% and 30.5\%), and white men $(16.1 \%$ and $40.7 \%)(P<0.0001)$. In multivariable-adjusted analyses, racial disparities in procedure utilization appeared somewhat larger and sex-based disparities remained significant. Unadjusted 30-day mortality after AMI in 1992 to 1993 for black women, black men, white women and white men was 20.4\%, 17.9\%, 23.1\% and $19.5 \%$, respectively $(P<0.0001)$; in 2009 to 2010 mortality was $17.1 \%, 15.3 \%, 18.2 \%$ and $16.2 \%$, respectively $(P<0.0001)$. In adjusted analyses, racial differences in mortality declined over time but differences by sex (higher mortality for women) persisted.
\end{abstract}

Conclusions: Disparities in AMl have declined modestly, but remain a problem, particularly with respect to patient sex. Keywords: Myocardial infarction, MI, Disparity, Outcomes, Race, Sex, Mortality, PCl, Hospitalization rates

\footnotetext{
* Correspondence: jasvinder.md@gmail.com

'Medicine Service, Birmingham Veterans Affairs Medical Center, the University of Alabama at Birmingham, 510 S 20th Street, Faculty Office Tower 805B, Birmingham, AL 35294, UK

Full list of author information is available at the end of the article
} 


\section{Background}

More than two decades of research has demonstrated significant disparities in the management of patients with acute myocardial infarction (AMI) based upon patient sex and race in both the United States (US) and Europe [1-5]. Reports from the US Institute of Medicine (IOM) and the European Union have called for providers and policymakers to confront and eliminate socioeconomic and racial and ethnic disparities in health care $[6,7]$. The World Health Organization has described the social determinants of health that lead to health inequities [8]. The US Affordable Care Act (ACA) established a dedicated office to reduce disparities [9].

While reduction of race- and sex-based disparities has been an international priority for decades, relatively few longitudinal studies have assessed the success of disparity reduction efforts. Moreover, most of the available studies were relatively short in duration (that is, 5 to 10 years) and few focused comprehensively on AMI $[1,3]$. In 2005, Jha et al. reported no decline in racial disparities for nine surgical procedures during the 1990s [10], but did not study disparities in AMI-associated hospitalization or mortality and this study has not been updated with contemporary data. The case fatality rate was consistently higher among Maori and Pacific Islands people than in Europeans in New Zealand for each age group and both sexes [5].

The overarching objective of our analysis was to comprehensively examine long-term trends in AMI disparities by patient race (black and white) and sex (male and female). In particular, we were interested in examining longitudinal differences in AMI-associated: 1) hospitalization rates; 2) utilization of revascularization procedures; and 3) mortality. In aggregate we felt that these three measures could comprehensively evaluate the full spectrum of AMI care from the underlying burden of cardiovascular disease (hospitalization rate), to processes of care (revascularization) and outcomes (mortality).

\section{Methods}

Data

We linked two US Medicare Provider Analysis and Review (MedPAR) Part A data files (one running from 1991 to 2005 and the second from 2006 to 2010) to identify fee-for-service beneficiaries hospitalized with AMI using International Classification of Diseases, Ninth Revision, Clinical Modification (ICD-9-CM) code, 410.xx. The MedPAR files contain administrative data for all feefor-service Medicare enrollees hospitalized in US hospitals including: demographics (age, sex, race/ethnicity); ICD-9$\mathrm{CM}$ codes for diagnoses and procedures; admission source (for example, emergency department or transfer from outside hospital); admission and discharge dates; discharge disposition (for example, home, nursing home, transfer to another acute-care hospital, dead); and death occurring up to three-years after discharge. The MedPAR files also include each patient's unique Medicare beneficiary number allowing for tracking of patients transferred between hospitals.

We applied several inclusion and exclusion criteria [see Additional file 1] to generate our final analytic cohort. We began by identifying all Non-Hispanic white or black patients hospitalized with an AMI during our study period. We focused our analysis on blacks and whites because the black/white racial designation has traditionally been more accurate than other racial designations and the number of patients of 'other' race/ethnicity categories were relatively small, particularly during the early 1990s [11].

We identified a 'new' AMI by limiting our analysis to each patient's first AMI hospitalization during a given one-year period by excluding index hospitalizations with prior AMI during the 12-months 'look back' preceding the index hospitalization. To implement the 'look back' we excluded patients $<66$ years of age at the time of their AMI and those lacking a full 12-months of MedPAR data preceding their AMI hospitalization; of necessity we excluded all patients hospitalized in 1991 and 2006 in order to allow us to apply the 'look back'. We excluded all AMI admissions occurring in 2006 because a lack of consistent patient identification numbers across our two MedPAR files precluded us from linking patients across the two data sets. To be consistent with prior studies focusing on AMI [12-14], we excluded patients: 1) enrolled in Medicare HMOs; 2) with a primary diagnoses of 410.X2 (indicating the subsequent episode of care for AMI); 3) patients discharged alive within one day of admission not against medical advice, as such patients likely represent miscoding of AMI. We also excluded AMI admissions after 1 December 2005 for the 1991 to 2005 data file and after 1 December 2010 for the 2006 to 2010 data file to ensure a 30-day follow-up window for each admission.

\section{Study outcomes}

We sought to evaluate changes in AMI disparities using an array of complementary measures, including hospitalization rates, receipt of revascularization procedures among those hospitalized and mortality [12-14]. Each measure was assessed in four discrete patient cohorts: black women, black men, white women, and white men. Revascularization included the receipt of three complementary cardiac procedures: 1) coronary angiography (with or without percutaneous coronary intervention (PCI); ICD-9-CM codes 37.21, 37.22, 37.23, 88.55, 88.56, 88.57); 2) PCI (ICD-9-CM codes 36.01, 36.02, 36.03, $36.04,36.05,36.06,36.07,36.09,00.66)$; and 3) coronary artery bypass surgery (CABG; ICD-9-CM codes 36.10, 
$36.11,36.12,36.13,36.14,36.15,36.16,36.17,36.18,36.19)$. We assessed receipt of each procedure at three different time-points: 1) during the index hospitalization (that is, at the admitting hospital); 2) during the index admission but after transfer from the admitting hospital to a second facility; and 3) within 30 days of AMI admission (which included procedures performed at the admitting hospital, after transfer, or during a subsequent readmission). Patients who underwent multiple procedures (for example, angiography plus $C A B G$ ) were included in the counts for each. If a patient had more than one of the same procedure (for example, two PCIs within 30 days), we only counted the first procedure. We examined unadjusted and adjusted mortality within 30 days of AMI admission using methods described below.

\section{Statistical analysis}

First, we compared the demographic characteristics and prevalence of key comorbid conditions by race and gender across the study period. We used analysis of variance (ANOVA) for comparisons of continuous variables and the chi-squared test for categorical variables. For simplicity, the study period was collapsed into two-year study increments (for example, 1992 to 1993, 1994 to 1995, and so on). Comorbid illnesses coded during the index admission were identified using algorithms developed by Elixhauser et al. [15].

Second, we compared unadjusted and standardized hospitalization rates for each of our four patient cohorts (black women, black men, white women and white men). AMI hospitalization rates were calculated as the number of AMI hospitalizations during a defined period of time in a given cohort (for example, 1992 to 1993) divided by the number of fee-for-service Medicare enrollees in that cohort during that period (for example, 1992 to 1993). We conducted comparisons across patient cohorts for a given time-period (for example, black women versus white women in 1992 to 1993) as well as longitudinal changes (for example, black women versus white women over the full study period) using statistical and graphical methods. We used analogous methods to compare differences in the utilization of cardiac procedures (catheterization, PCI and CABG) across patient cohorts (for example, black women compared to white women during a fixed time-period (1992 to 1993)) as well as to compare longitudinal changes over time.

Third, we examined trends in unadjusted and riskadjusted 30-day mortality using hierarchical generalized linear models. Our models were based upon models developed by the US Centers for Medicare and Medicaid Services and adjusted for patient demographics and comorbidities and accounted for clustering of patients within hospitals [16]. For 30-day mortality, rates were adjusted for age, year, location of MI, unstable angina, chronic atherosclerosis, cardiopulmonary-respiratory failure and shock, valvular heart disease, hypertension, stroke, cerebrovascular disease, renal failure, COPD, pneumonia, diabetes, protein-calorie malnutrition, dementia, hemiplegia, paraplegia, paralysis, functional disability, peripheral vascular disease, metastatic cancer, trauma in last year, major psychiatric disorders, chronic liver disease, history of PCI, history of CABG and history of heart failure.

Finally, we conducted an array of sensitivity analyses. First, we repeated our analyses of procedure utilization using an endpoint of 30-day revascularization (PCI or CABG) in place of our focus on individual procedures. Second, we repeated our evaluations of procedure utilization using models that adjusted for patient age and comorbidities and accounted for clustering of patients within hospitals; these models allowed us to examine whether differences in utilization might be explained by differences in patient comorbidity and/or differences in the hospitals where each patient cohort received care. Third, we repeated our analyses without applying the oneyear look-back, which allowed us to include patients from 1991 and 2006 in our analyses. Fourth, in order to explore differences in the timing of revascularization, we examined the proportion of patients who received a procedure during the index hospitalization, after transfer to another acute care hospital, and during a separate hospital admission but within 30-days of the index admission in each of our four patient cohorts. All sensitivity analyses and model details are available by request. All analyses were performed using SAS Version 9.2 (Cary, NC, USA). The University of Iowa Institutional Review Board approved this study.

\section{Results}

\section{Cohort characteristics}

Our study consisted of 4,045,267 AMI admissions between 1992 and 2010 [see Additional file 1] of which 166,660 admissions were black women, 116,201 black men, 1,870,816 white women and 1,891,590 were white men. Black and white women hospitalized with AMI were consistently older than black and white men (Table 1). Among each of the four patient cohorts, age increased by approximately three-years over the study period (Table 1).

\section{AMI hospitalization rates}

The per-capita hospitalization rate for AMI (number of hospitalizations during a specific year/number of Medicare enrollees in that specific patient group) differed markedly across the four study cohorts (Table 1 and Figure 1A), although there was evidence that the disparities narrowed over time. In particular during the early 1990s, white men had a higher hospitalization rate and white women a lower hospitalization rate $(P<0.001)$. Despite narrowing of the differences between cohorts over time, disparities in AMI 
Table 1 Characteristics of black and white men and women hospitalized with acute myocardial infarction (AMI)

\begin{tabular}{|c|c|c|c|c|}
\hline & Black women & Black men & White women & White men \\
\hline \multicolumn{5}{|c|}{ Hospitalizations, number (rate per 10,000 Medicare enrollees) ${ }^{a}$} \\
\hline 1992-1993 & $16237(61.6)$ & $11958(73.2)$ & $219273(72.0)$ & $228751(113.2)$ \\
\hline 1994-1995 & $17774(63.4)$ & $12889(75.6)$ & $227347(72.4)$ & $237520(112.9)$ \\
\hline 1996-1997 & $18541(64.9)$ & $13351(77.3)$ & $228918(72.5)$ & $235828(110.3)$ \\
\hline 1998-1999 & $19562(67.3)$ & $13167(75.0)$ & $226734(71.5)$ & $226984(104.5)$ \\
\hline 2000-2001 & $21100(70.5)$ & $14091(77.5)$ & $231992(72.6)$ & $226802(102.4)$ \\
\hline $2002-2003$ & $21918(71.7)$ & $14595(77.8)$ & $228463(71.5)$ & $223957(99.3)$ \\
\hline $2004-2005^{b}$ & $18276(59.3)$ & $12743(65.7)$ & $190021(59.9)$ & $187818(81.5)$ \\
\hline 2007-2008 & $17043(49.3)$ & $11961(54.3)$ & $169446(49.9)$ & $170004(67.2)$ \\
\hline 2009-2010 & $16209(43.1)$ & $11446(46.7)$ & $148622(41.0)$ & $153926(55.6)$ \\
\hline \multicolumn{5}{|c|}{ Age, mean (standard deviation) } \\
\hline 1992-1993 & $77.1(7.6)$ & $75.1(6.8)$ & $78.4(7.4)$ & $75.5(6.7)$ \\
\hline 1994-1995 & $77.3(7.7)$ & $75.3(6.9)$ & $78.7(7.5)$ & $75.8(6.8)$ \\
\hline 1996-1997 & $77.8(7.8)$ & $75.7(7.1)$ & $79.4(7.6)$ & $76.4(7.0)$ \\
\hline 1998-1999 & $78.6(8.0)$ & $76.1(7.2)$ & $80.1(7.7)$ & $77.1(7.1)$ \\
\hline 2000-2001 & $79.0(8.2)$ & $76.5(7.4)$ & $80.6(7.8)$ & $77.5(7.3)$ \\
\hline 2002-2003 & $79.2(8.3)$ & $76.4(7.5)$ & $80.9(7.9)$ & $77.7(7.4)$ \\
\hline 2004-2005 & $79.7(8.3)$ & $76.6(7.5)$ & $81.2(8.0)$ & $78.1(7.5)$ \\
\hline 2007-2008 & $79.8(8.7)$ & $76.5(7.7)$ & $81.5(8.3)$ & $78.1(7.9)$ \\
\hline 2009-2010 & $79.7(8.7)$ & $76.3(7.8)$ & $81.6(8.4)$ & $78.1(8.1)$ \\
\hline \multicolumn{5}{|c|}{ Comorbidity, number $(\%)^{c}$} \\
\hline \multicolumn{5}{|c|}{ Diabetes } \\
\hline 1992-1993 & $5671(34.9)$ & $2855(23.9)$ & $50955(23.2)$ & $44725(19.6)$ \\
\hline 1994-1995 & $6975(39.2)$ & $3648(28.3)$ & $58780(25.9)$ & $53720(22.6)$ \\
\hline 1996-1997 & $7700(41.5)$ & $4004(30.0)$ & $60433(26.4)$ & $56631(24.0)$ \\
\hline 1998-1999 & $7993(40.9)$ & $4146(31.5)$ & $60627(26.7)$ & $57138(25.2)$ \\
\hline 2000-2001 & 8639 (40.9) & $4608(33.7)$ & $61893(26.7)$ & $59138(26.1)$ \\
\hline $2002-2003$ & 8646 (39.5) & $4815(33.0)$ & $59646(26.1)$ & $58803(26.3)$ \\
\hline 2004-2005 & $7021(38.4)$ & $4171(32.7)$ & $48017(25.3)$ & $48774(26.0)$ \\
\hline 2007-2008 & $6262(36.7)$ & $3791(31.7)$ & $40832(24.1)$ & $43512(25.6)$ \\
\hline 2009-2010 & $5867(36.2)$ & $3589(31.4)$ & $35738(24.1)$ & $39389(25.6)$ \\
\hline \multicolumn{5}{|c|}{ Congestive heart failure, number $(\%)^{c}$} \\
\hline 1992-1993 & $7662(47.2)$ & 4895 (40.9) & 98207 (44.8) & 86088 (37.6) \\
\hline 1994-1995 & 8433 (47.4) & $5403(41.9)$ & $103382(45.5)$ & 89728 (37.8) \\
\hline 1996-1997 & 9149 (49.3) & $5928(44.4)$ & 105829 (46.2) & 91840 (38.9) \\
\hline 1998-1999 & $9883(50.5)$ & $5897(44.8)$ & $106226(46.8)$ & 89515 (39.4) \\
\hline 2000-2001 & $10501(49.8)$ & 6335 (45.0) & $107129(46.2)$ & 88753 (39.1) \\
\hline 2002-2003 & $10892(49.7)$ & $6565(45.0)$ & $107208(46.9)$ & 89274 (39.9) \\
\hline 2004-2005 & 9375 (51.3) & $5775(45.3)$ & $91500(48.2)$ & 77046 (41.0) \\
\hline 2007-2008 & 7989 (46.9) & 5189 (43.4) & 75421 (44.5) & 63959 (37.6) \\
\hline 2009-2010 & 7439 (45.9) & $4817(42.1)$ & $64552(43.4)$ & 56955 (37.0) \\
\hline \multicolumn{5}{|c|}{ Obesity, number (\%) } \\
\hline 1992-1993 & 375 (2.3) & $101(0.8)$ & $3888(1.8)$ & $2752(1.2)$ \\
\hline 1994-1995 & 609 (3.4) & $176(1.4)$ & $5458(2.4)$ & 3899 (1.6) \\
\hline
\end{tabular}


Table 1 Characteristics of black and white men and women hospitalized with acute myocardial infarction (AMI) (Continued)

\begin{tabular}{lllll}
\hline $1996-1997$ & $696(3.8)$ & $190(1.4)$ & $6194(2.7)$ & $4695(2.0)$ \\
$1998-1999$ & $701(3.6)$ & $227(1.7)$ & $6172(2.7)$ & $4873(2.2)$ \\
$2000-2001$ & $825(3.9)$ & $257(1.8)$ & $6540(2.8)$ & $5322(2.4)$ \\
$2002-2003$ & $954(4.4)$ & $298(2.0)$ & $6528(2.9)$ & $5820(2.6)$ \\
$2004-2005$ & $834(4.6)$ & $286(2.2)$ & $5860(3.1)$ & $5410(2.9)$ \\
$2007-2008$ & $819(4.8)$ & $313(2.6)$ & $5478(3.2)$ & $5802(3.4)$ \\
$2009-2010$ & $864(5.3)$ & $353(3.1)$ & $5329(3.6)$ & $6009(3.9)$ \\
\hline
\end{tabular}

${ }^{a}$ The denominator is all traditional Medicare enrollees in each stratum, that is, black female, black male, white female, and white male; ${ }^{\mathrm{b}}$ patients from calendar year 2006 were excluded as detailed in the Methods section; ' The denominator is AMI patients in each stratum, that is, black female, black male, white female, and white male.

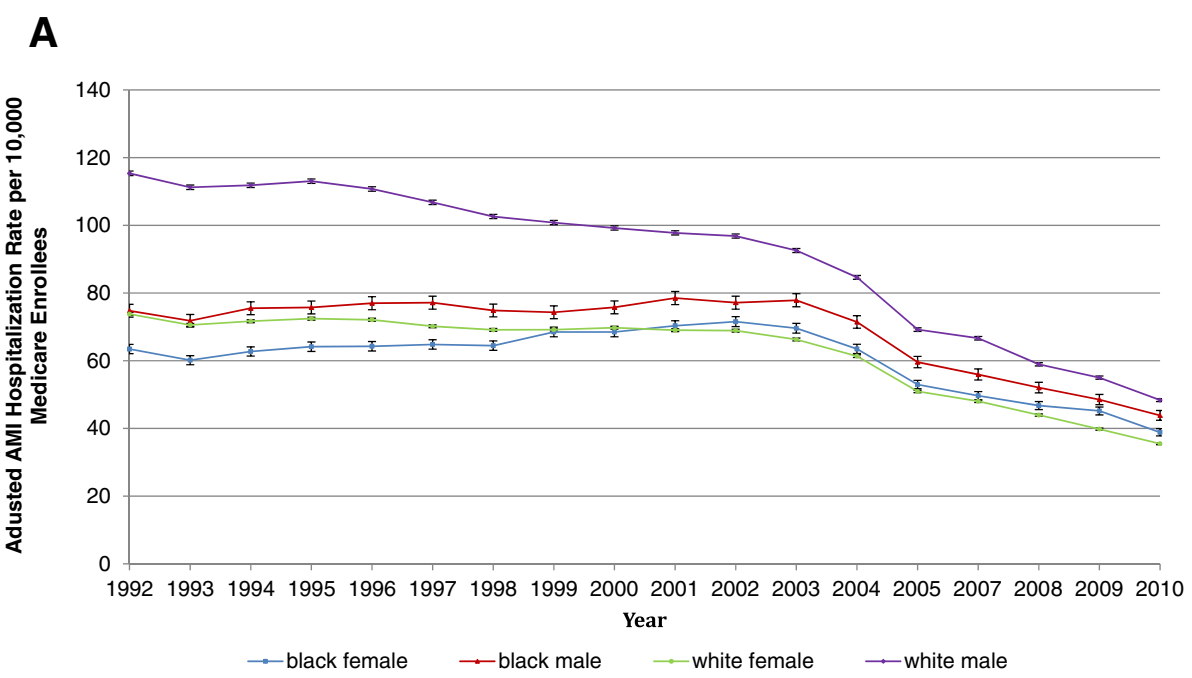

B

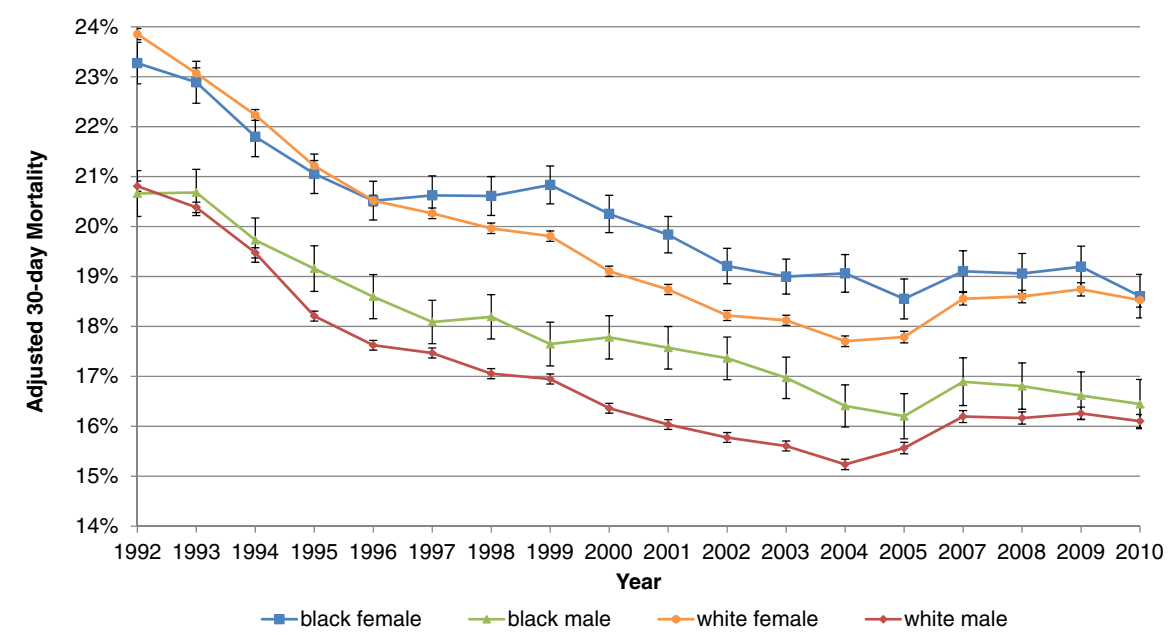

Figure 1 Adjusted hospitalization rates for AMI (A) and 30-day all-cause mortality after admission for AMI (B). Y-axis shows adjusted AMI hospitalization per 10,000 Medicare enrollees; rates were adjusted for age and year (A). For 30-day all-cause mortality, rates were adjusted for age, year, location of $\mathrm{Ml}$, unstable angina, chronic atherosclerosis, cardiopulmonary-respiratory failure and shock, valvular heart disease, hypertension, stroke, cerebrovascular disease, renal failure, COPD, pneumonia, diabetes, protein-calorie malnutrition, dementia, hemiplegia, paraplegia, paralysis, functional disability, peripheral vascular disease, metastatic cancer, trauma in last year, major psychiatric disorders, chronic liver disease, history of $\mathrm{PCl}$, history of $\mathrm{CABG}$ and history of heart failure (B). AMI, acute myocardial infarction; CABG, coronary artery bypass surgery; COPD, chronic obstructive pulmonary disease; $\mathrm{PCl}$, percutaneous coronary intervention. 
hospitalization rates by race and sex remained statistically significant in 2009 to $2010(P<0.001)$. Viewed from an alternate perspective, each cohort experienced significant decreases in AMI hospitalization rates between 1992 to 1993 and 2009 to 2010 ( $P<0.01$ for each cohort over time).

\section{Procedure utilization}

Unadjusted rates of all procedures (coronary angiography, PCI and CABG) within 30 days of AMI admission were lower for blacks compared to whites and women compared to men throughout the study period (Table 2 and Figures 2A, B, and C). For example, in 1992 to 1993 $29.0 \%$ of black women and $36.5 \%$ of black men underwent coronary angiography (with or without PCI) within 30 days of AMI admission as compared to $34.3 \%$ of white women and $46.7 \%$ of white men $(P<0.0001)$. Likewise, in 2009 to $2010,5.1 \%$ of black women and $8.3 \%$ of black men received CABG within 30 days of AMI admission compared to $5.9 \%$ of white women and $11.6 \%$ of white men $(P<0.0001)$. Viewed longitudinally, all patient cohorts saw an approximately $20 \%$ rise in the percentage of patients who received coronary angiography within 30 days of AMI admission over the study period (Table 2 and Figures 2A, B, and C). In addition, the proportion of these procedures that were performed during the index admission increased over time for all patient cohorts [see Additional files 2, 3 and 4].

In analyses that adjusted for patient age and comorbidity [see Additional file 5] and additionally accounting for clustering of patients within hospitals [see Additional file 6], results were somewhat different. In particular, we found that the lower procedure utilization in blacks as compared to whites was largely eliminated after accounting for differences in age and comorbidity [see Additional file 5] although disparities by sex (lower procedure utilization among women as compared to men) persisted. In analyses that also accounted for clustering of patients within hospitals, racial disparities in procedure utilization appeared somewhat larger and sex-based disparities remained significant [see Additional file 6].

\section{Mortality}

Unadjusted mortality within 30 days of admission was lower for blacks than for whites and for men than for women throughout the study period (Table 3), although differences appeared to decline over time. For example, in 1992 to 1993, the 30-day mortality rate after index AMI was lower in black men compared to white men (17.9\% versus $19.5 \% ; P \leq 0.0001)$ and in black women compared to white women $(20.4 \%$ versus $23.1 \%$; $P<0.0001)$. By 2009 to 2010, absolute differences between groups had narrowed, but remained significant (Table 3). In analyses that adjusted for differences in demographics and comorbidity (Figure 1B), racial differences in mortality declined over time but differences by sex (higher mortality for women) persisted. Viewed from a different perspective 30-day mortality after AMI decreased significantly $(P<0.0001)$ within each cohort over the study period.

\section{Discussion}

In a longitudinal analysis of US Medicare beneficiaries hospitalized with AMI between 1992 and 2010, we report a number of important trends in disparities. We found that AMI hospitalization rates declined substantially for all patient groups. We also found that differences in hospitalization rates in blacks and whites and men and women have narrowed over time, but disparities persist. Utilization of interventional procedures after AMI has increased over time, but disparities persist with both women and blacks being significantly less likely to receive most procedures. Lastly, 30-day mortality following an AMI has declined over time for all patient groups, but remains significantly higher for women compared to men. Several findings merit further discussion.

First, we found declines in overall hospitalization rates for AMI among all four of our study cohorts (black women, black men, white women, and white men). While there are several studies reporting changes over time in AMI mortality or quality of AMI care [17-19], there are fewer studies of long-term trends in AMI hospitalization rates, with somewhat contradictory results. Chen and Krumholz examined trends in AMI hospitalization among Medicare beneficiaries between 2002 and 2007 and noted an approximately $20 \%$ decline in hospitalization rates [20]; they also found evidence of higher AMI hospitalization rates among men compared to women and white men as compared to black men. Similarly, Talbott et al. reported a $20 \%$ decline in AMI hospitalization rates using the Centers for Disease Control (CDC) data [21]. Alternatively, data show declines in cardiovascular disease mortality over time without corresponding reductions in AMI hospitalization rates [22]. Our findings are particularly intriguing when viewed through the lens of increasingly sensitive diagnostic tests for AMI (that is, serum troponin) that would be expected to increase AMI diagnosis and hospitalization rates [23]. In a nationwide Danish study, the standardized incidence rate per 100,000 people decreased in the 25-year period, 1984 to 2008 , by $37 \%$ for women (from 209 to 131 ) and by $48 \%$ for men (from 410 to 213) [24].

Our finding of differential hospitalization rates by race and sex is noteworthy and merits brief comment. It is possible that the lower hospitalization rates truly represent lower population-based AMI risk among blacks (compared to whites) and women (compared to men). However, an array of epidemiological data suggests a higher burden of cardiovascular disease among blacks making this scenario unlikely $[25,26]$. An alternative and more ominous 
Table 2 Percentage of black and white men and women who underwent selected procedures within 30 days of admission for AMI

\begin{tabular}{|c|c|c|c|c|}
\hline & Black women & Black men & White women & White men \\
\hline \multicolumn{5}{|c|}{ Coronary angiography, number (\%) } \\
\hline \multicolumn{5}{|c|}{ Number (\%) who underwent the procedure within 30 days of admission ${ }^{a}$} \\
\hline 1992-1993 & $4707(29.0)$ & $4362(36.5)$ & $75134(34.3)$ & $106829(46.7$ \\
\hline 1994-1995 & $6169(34.7)$ & $5391(41.8)$ & $89097(39.2)$ & $123998(52.2$ \\
\hline 1996-1997 & $7101(38.3)$ & $6086(45.6)$ & $98228(42.9)$ & $131268(55.7$ \\
\hline 1998-1999 & $7563(38.7)$ & $6209(47.2)$ & $99993(44.1)$ & $128880(56.8$ \\
\hline 2000-2001 & $8565(40.6)$ & 6955 (49.4) & $107484(46.3)$ & 134706 (59.4 \\
\hline 2002-2003 & 9623 (43.9) & 7757 (53.2) & 112046 (49.0) & $139416(62.3$ \\
\hline 2004-2005 & 8356 (45.7) & 7168 (56.3) & $97082(51.1)$ & $120720(64.3$ \\
\hline 2007-2008 & 7799 (45.8) & $6883(57.6)$ & 87771 (51.8) & $110588(65.1$ \\
\hline 2009-2010 & 7867 (48.5) & $6768(59.1)$ & $78727(53.0)$ & $102085(66.3$ \\
\hline
\end{tabular}

Among those who underwent angiography, number (\%) who received their angiography during the index admission ${ }^{\mathbf{b}}$

\begin{tabular}{|c|c|c|c|c|}
\hline 1992-1993 & $3456(73.4)$ & $3193(73.2)$ & $46938(62.5)$ & $68346(64.0)$ \\
\hline 1994-1995 & 4544 (73.7) & $4013(74.4)$ & $55723(62.5)$ & $80312(64.8)$ \\
\hline 1996-1997 & $5260(74.1)$ & $4500(73.9)$ & 63176 (64.3) & $86863(66.2)$ \\
\hline 1998-1999 & $5560(73.5)$ & $4674(75.3)$ & $66205(66.2)$ & 87498 (67.9) \\
\hline $2000-2001$ & 6486 (75.7) & $5245(75.4)$ & 73240 (68.1) & 94081 (69.8) \\
\hline $2002-2003$ & 7465 (77.6) & $6074(78.3)$ & 80892 (72.2) & 103264 \\
\hline 2004-2005 & 6759 (80.9) & 5869 (81.9) & $74730(77.0)$ & 95221 (78.9) \\
\hline $2007-2008$ & 6521 (83.6) & $5826(84.6)$ & $72270(82.3)$ & 93147 (84.2) \\
\hline 2009-2010 & 6791 (86.3) & $5887(87.0)$ & 66669 (84.7) & 88275 (86.5) \\
\hline
\end{tabular}

Percutaneous coronary intervention (PCl), number (\%)

Number (\%) who underwent the procedure within 30 days of index AMI admission ${ }^{\mathrm{a}}$

$\begin{array}{lllll}1992-1993 & 1394(8.6) & 1243(10.4) & 28013(12.8) & 36743(16.1) \\ 1994-1995 & 2009(11.3) & 1768(13.7) & 36406(16.0) & 47057(19.8) \\ 1996-1997 & 2483(13.4) & 2120(15.9) & 42885(18.7) & 53486(22.7) \\ 1998-1999 & 2861(14.6) & 2335(17.7) & 47506(21.0) & 58746(25.9) \\ 2000-2001 & 3398(16.1) & 3019(21.4) & 53981(23.3) & 66189(29.2) \\ 2002-2003 & 4134(18.9) & 3633(24.9) & 59331(26.0) & 73620(32.9) \\ 2004-2005 & 3956(21.7) & 3602(28.3) & 54776(28.8) & 68973(36.7) \\ 2007-2008 & 3748(22.0) & 3564(29.8) & 49938(29.5) & 65672(38.6) \\ 2009-2010 & 3916(24.2) & 3728(32.6) & 45375(30.5) & 62705(40.7)\end{array}$

Among those who underwent $\mathrm{PCl}$, number (\%) during the index admission ${ }^{\mathrm{b}}$

$\begin{array}{lllll}1992-1993 & 857(61.5) & 754(60.7) & 14567(52.0) & 19915(54.2) \\ 1994-1995 & 1241(61.8) & 1120(63.4) & 19519(53.6) & 26582(56.5) \\ 1996-1997 & 1600(64.4) & 1329(62.7) & 24381(56.9) & 31498(58.9) \\ 1998-1999 & 1841(64.4) & 1497(64.1) & 28156(59.3) & 35960(61.2) \\ 2000-2001 & 2330(68.6) & 2026(67.1) & 33527(62.1) & 42653(64.4) \\ 2002-2003 & 2916(70.5) & 2607(71.8) & 40308(67.9) & 51497(70.0) \\ 2004-2005 & 2972(75.1) & 2802(77.8) & 40402(73.8) & 52304(75.8) \\ 2007-2008 & 2999(80.0) & 2926(82.1) & 40387(80.9) & 54283(82.7) \\ 2009-2010 & 3315(84.7) & 3148(84.4) & 38285(84.4) & 53819(85.8)\end{array}$


Table $\mathbf{2}$ Percentage of black and white men and women who underwent selected procedures within $\mathbf{3 0}$ days of admission for AMI (Continued)

Coronary artery bypass grafting (CABG), number (\%)

Number (\%) who underwent the procedure within 30 days of index AMI admission ${ }^{\mathrm{a}}$

$\begin{array}{lllll}1992-1993 & 1011(6.2) & 919(7.7) & 19058(8.7) & 33508(14.7) \\ 1994-1995 & 1277(7.2) & 1254(9.7) & 23047(10.1) & 39739(16.7) \\ 1996-1997 & 1453(7.8) & 1402(10.5) & 25006(10.9) & 41789(17.7) \\ 1998-1999 & 1483(7.6) & 1329(10.1) & 22626(10.0) & 37796(16.7) \\ 2000-2001 & 1603(7.6) & 1461(10.4) & 21567(9.3) & 36690(16.2) \\ 2002-2003 & 1569(7.2) & 1547(10.6) & 19654(8.6) & 34226(15.3) \\ 2004-2005 & 1160(6.4) & 1250(9.8) & 14560(7.7) & 26342(14.0) \\ 2007-2008 & 937(5.5) & 1064(8.9) & 11125(6.6) & 21249(12.5) \\ 2009-2010 & 830(5.1) & 954(8.3) & 8806(5.9) & 17773(11.6)\end{array}$

Among those who underwent CABG, number (\%) during the index admission ${ }^{\text {b }}$

\begin{tabular}{|c|c|c|c|c|}
\hline 1992-1993 & $519(51.3)$ & $435(47.3)$ & 7747 (40.7) & $14152(42.2)$ \\
\hline 1994-1995 & $624(48.9)$ & 647 (51.6) & 9537 (41.4) & $17294(43.5)$ \\
\hline 1996-1997 & $713(49.1)$ & $729(52.0)$ & $10868(43.5)$ & $19127(45.8)$ \\
\hline 1998-1999 & 766 (51.7) & 703 (52.9) & $10541(46.6)$ & $18400(48.7)$ \\
\hline $2000-2001$ & $871(54.3)$ & $770(52.7)$ & $10617(49.2)$ & $18859(51.4)$ \\
\hline $2002-2003$ & 913 (58.2) & 905 (58.5) & $10726(54.6)$ & $19471(56.9)$ \\
\hline 2004-2005 & $704(60.7)$ & $787(63.0)$ & 8822 (60.6) & $16693(63.4)$ \\
\hline 2007-2008 & $604(64.5)$ & $697(65.5)$ & 7369 (66.2) & $14595(68.7)$ \\
\hline 2009-2010 & $565(68.1)$ & $648(67.9)$ & $5883(66.8)$ & 12332 (69.4) \\
\hline
\end{tabular}

Revascularization (CABG or $\mathrm{PCI})$, number (\%)

Number (\%) who underwent the procedure within 30 days of index AMI admission ${ }^{\mathrm{a}}$

$\begin{array}{lllll}1992-1993 & 2325(14.3) & 2108(17.6) & 45598(20.8) & 67970(29.7) \\ 1994-1995 & 3211(18.1) & 2943(22.8) & 57580(25.3) & 84056(35.4) \\ 1996-1997 & 3864(20.8) & 3454(25.9) & 66268(29.0) & 92920(39.4) \\ 1998-1999 & 4269(21.8) & 3604(27.4) & 68755(30.3) & 94321(41.6) \\ 2000-2001 & 4921(23.3) & 4413(31.3) & 74229(32.0) & 100637(44.4) \\ 2002-2003 & 5606(25.6) & 5080(34.8) & 77563(34.0) & 105419(47.1) \\ 2004-2005 & 5028(27.5) & 4758(37.3) & 68167(35.9) & 93067(49.6) \\ 2007-2008 & 4598(27.0) & 4540(38.0) & 59979(35.4) & 84696(49.8) \\ 2009-2010 & 4685(28.9) & 4605(40.2) & 53326(35.9) & 78561(51.0)\end{array}$

Among those who underwent revascularization, number (\%) during the index admission ${ }^{\mathbf{b}}$

$\begin{array}{lllll}1992-1993 & 1336(57.5) & 1157(54.9) & 21524(47.2) & 32793(48.3) \\ 1994-1995 & 1820(56.7) & 1712(58.2) & 28006(48.6) & 42250(50.3) \\ 1996-1997 & 2268(58.7) & 2014(58.3) & 34286(51.7) & 49217(53.0) \\ 1998-1999 & 2570(60.2) & 2163(60.0) & 37872(55.1) & 53006(56.2) \\ 2000-2001 & 3156(64.1) & 2754(62.4) & 43410(58.5) & 60168(59.8) \\ 2002-2003 & 3769(67.2) & 3446(67.8) & 50186(64.7) & 69457(65.9) \\ 2004-2005 & 3630(72.2) & 3522(74.0) & 48465(71.1) & 67455(72.5) \\ 2007-2008 & 3541(77.0) & 3568(78.6) & 47008(78.4) & 67286(79.4) \\ 2009-2010 & 3837(81.9) & 3741(81.2) & 43575(81.7) & 64784(82.5)\end{array}$

${ }^{\mathrm{a}}$ The denominator is AMI patients in each stratum, that is, black women, black men, white women and white men; ${ }^{\mathrm{b}}$ the denominator is AMI patients who underwent the respective procedure in each stratum, that is, black women, black men, white women and white men. AMI, acute myocardial infarction; CABG, coronary artery bypass surgery; $\mathrm{PCl}$, percutaneous coronary intervention. 

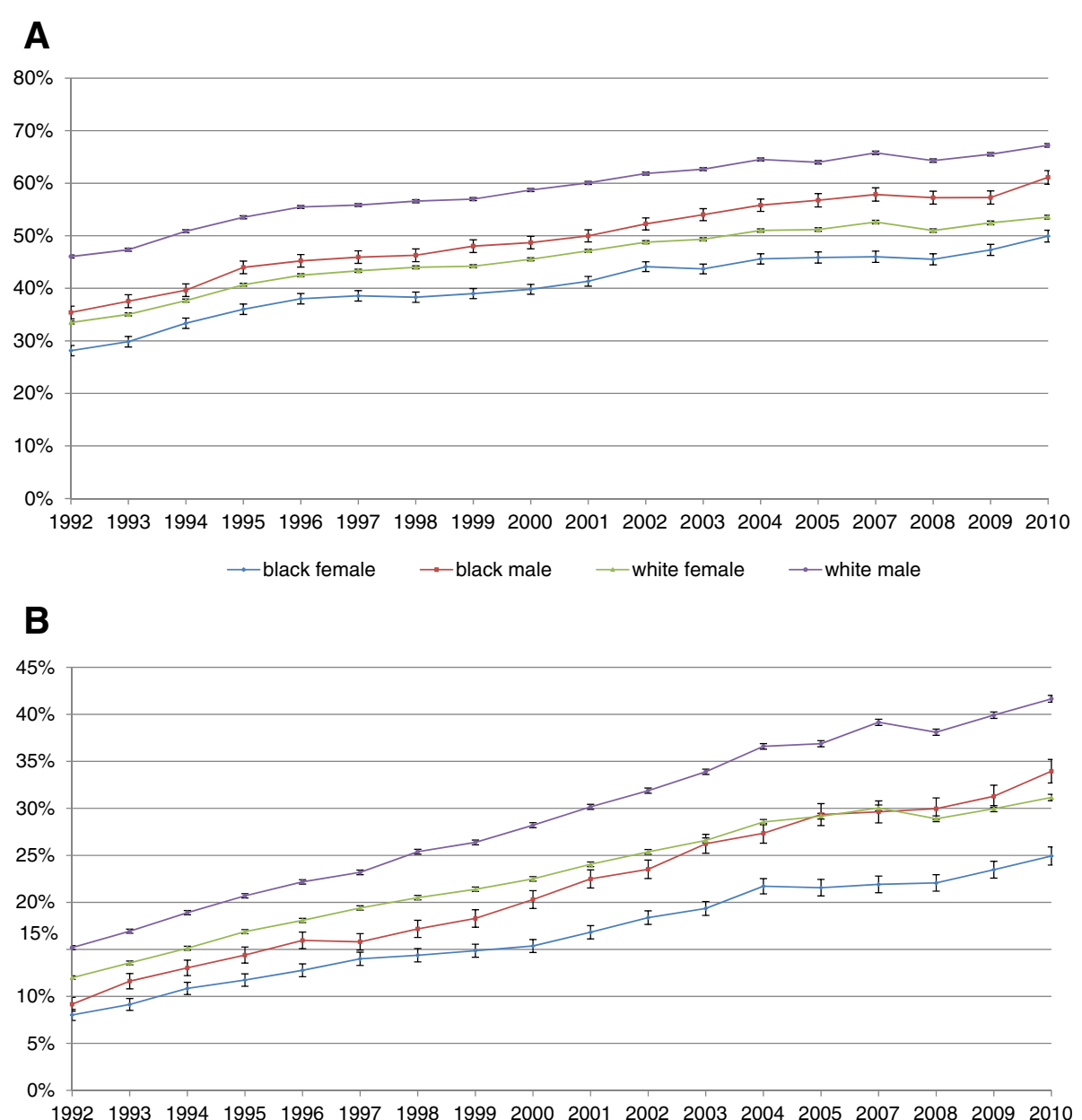

$\rightarrow$ black female $\rightarrow$ black male $\quad \rightarrow$ white female $\quad \rightarrow$ white male

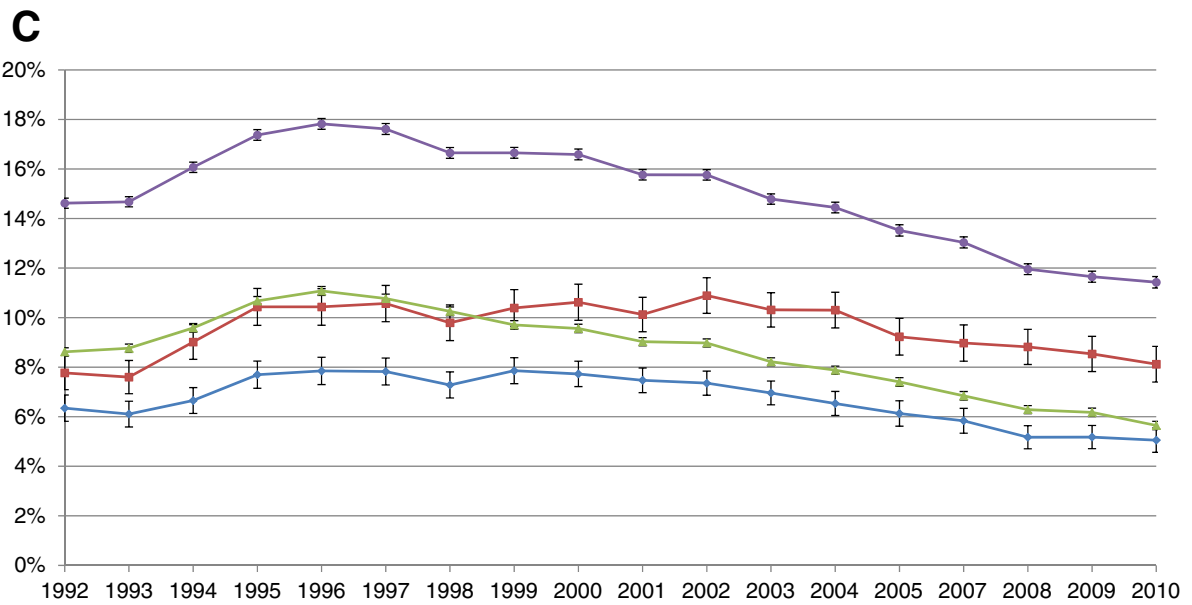

$\rightarrow$ black female $\rightarrow$-black male $\rightarrow$ white female $\rightarrow$-wite male

Figure 2 Unadjusted utilization of coronary angiography (A), PCI (B) and CABG (C) within $\mathbf{3 0}$ days after admission for AMI. Y-axis shows the unadjusted percentage of Medicare beneficiaries hospitalized with AMI receiving angiography (with or without revascularization) (A), PCl (B) or CABG (C) within 30 days of admission. AMl, acute myocardial infarction; CABG, coronary artery bypass surgery; PCl, percutaneous coronary intervention. 
Table 3 Percentage ${ }^{a}$ of black and white men and women who died within $\mathbf{3 0}$ days after admission for AMI (unadjusted estimates)

\begin{tabular}{lllll}
\hline & Black women & Black men & White women & White male \\
\hline Mortality, number (\%) & & & & \\
$1992-1993$ & $3311(20.4)$ & $2145(17.9)$ & $50641(23.1)$ & $44578(19.5)$ \\
$1994-1995$ & $3492(19.7)$ & $2189(17.0)$ & $50269(22.1)$ & $43655(18.4)$ \\
$1996-1997$ & $3512(18.9)$ & $2139(16.0)$ & $48151(21.0)$ & $41907(17.8)$ \\
$1998-1999$ & $3761(19.2)$ & $2213(16.8)$ & $46539(20.5)$ & $40065(17.7)$ \\
$2000-2001$ & $3952(18.7)$ & $2361(16.8)$ & $45521(19.6)$ & $38349(16.9)$ \\
$2002-2003$ & $3847(17.6)$ & $2396(16.4)$ & $42912(18.8)$ & $36805(16.4)$ \\
$2004-2005$ & $3122(17.1)$ & $1921(15.1)$ & $34193(18.0)$ & $29289(15.6)$ \\
$2007-2008$ & $2751(16.1)$ & $1790(15.0)$ & $29842(17.6)$ & $26226(15.4)$ \\
$2009-2010$ & $2768(17.1)$ & $1751(15.3)$ & $26994(18.2)$ & $24889(16.2)$ \\
\hline
\end{tabular}

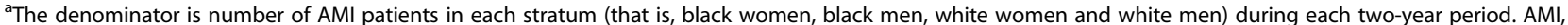
acute myocardial infarction.

explanation is that the lower hospitalization rates reflect under-diagnosis of AMI in blacks and women in the pre-hospital setting. Differences in AMI hospitalization rates by race and sex remained statistically significant in 2009 to $2010(P<0.001)$ despite narrowing of the differences between cohorts over time in analyses adjusted for age and year (Figure 1A). This implies that only some racial and sex differences were attributable to age and period effects, and some disparities still persisted, although they narrowed in adjusted analyses. Another study using the CDC data reported that the male-female ratio for AMI hospitalization rates remained constant at 2:1 from 2000 to 2008 [21].

Second, we found a marked increase in the utilization of revascularization among all patient cohorts over time. These results are consistent with prior studies [22]. Interestingly, while disparities in revascularization have been well documented [27], relatively few studies have examined whether disparities in revascularization have declined over time [28]. Peterson et al., using data from the National Registry of Myocardial Infarction (NRMI) found evidence that disparities by race and sex in the use of revascularization procedures did not decline between 1990 and 2006 [18]. Alternatively, Jha and colleagues found evidence that disparities for certain revascularization procedures declined among certain patient populations between 1992 and 2001 (for example, PCI in black and white women) but other disparities persisted (for example, CABG in black and white men) [10]. Our results extend prior studies by examining a more comprehensive list of revascularization procedures over a longer time period. Our unadjusted analyses showed minimal reductions in disparities for most procedures and that disparities between men and women remain particularly large. Interestingly, in analyses that adjusted for patient comorbidity and clustering of patients within hospitals [see Additional file 1], racial disparities appeared small although disparities by patient sex remained significant. The differences between our unadjusted and adjusted results are consistent with prior studies that have suggested that many disparities can be explained by differences in patients' acuity and comorbidity and also the quality and practice patterns of the physicians and hospitals where different patient groups seek care [2,18,29,30].

Third, our findings with regards to mortality are important and extend prior work. We found that racial disparities in AMI 30-day mortality grew relatively larger in the early part of the $21^{\text {st }}$ century, but have declined over the last five years. Alternatively, disparities by patient sex (higher mortality for both black and white women when compared to their male counterparts) have declined little [31]; higher mortality has been reported in younger ( $<50$ years), but not older women compared to men of the same age after AMI [32]. Studies have suggested that sexbased disparities in mortality can be attributed in part to differences in the receipt of evidence-based treatments and interventions, but also perhaps to differences in AMI presentation, severity and age-of-onset [33]. Irrespective of the exact cause, the sex-based differences in AMI mortality are striking, similar to a previous population-based Finnish study [34]. In this study including all age groups comparing 1994 to 1996 to 2000 to 2002 in two registers, the 28-day case fatality after AMI declined in men from $46.5 \%$ to $41.0 \%$ in the FINAMI register and from $54.7 \%$ to $50.1 \%$ in the Finnish National Cardiovascular Disease Register (CVDR), respectively. The corresponding numbers in women decreased from $53.5 \%$ to $48.6 \%$ in FINAMI and from $58.9 \%$ to $54.6 \%$ in CVDR [34]. Another recent study found that mortality from ischemic heart disease increased across all ages in adult women whereas the corresponding increase was blunted at older ages in men [35], a potential explanation of sex differences in AMI mortality in our data as well. A Dutch study of 14,434 patients in coronary units from 1985 to 2008 showed no 
sex difference in adjusted 30-day mortality [36], as well as a decline in mortality in both men and women over a 24-year period. The differences in country setting, health care delivery system, patient comorbidity load and single center versus national data between this previous study and our study may explain the differences in findings. Our mortality models were based on models developed by the US Centers for Medicare and Medicaid Services and adjusted for hospital clustering and, therefore, our confidence in these findings is high.

Our study has several limitations that warrant mention. First, our analysis was limited to fee-for-service Medicare beneficiaries and should be extended to other populations with caution. In particular, it is uncertain whether an evaluation of a younger non-Medicare population would yield similar results. Second, our analysis focused exclusively on AMI and we cannot comment on long-term trends in disparities for other conditions. Third, our analyses could have been impacted by changes in coding over time related to either AMI diagnosis or procedures. However, there is a long history of using Medicare data in the evaluation of AMI and we are unaware of any coding changes that would have directly impacted our results. Fourth, Medicare data may have race misclassification since this was not self-identified prospectively; however, this would bias our findings towards null and, therefore, our findings are conservative. In addition, this is a general limitation of studies using Medicare data. The impact of the exclusion of 2006 is unclear; however we have a longenough observation period, and a single year is unlikely to have meaningfully changed the findings. Fifth, the timetrends analyses for where the procedures were done are unadjusted (index hospitalization, transfer hospitalization, or post-discharge within 30 days); it is anticipated that a reduction in the length of AMI stay over the study period likely impacted these rates, and, therefore, these should be interpreted with caution. Lastly, we are unable to account for patient preferences, which play a role in utilization of these procedures or physician preferences, or the clinical information about the appropriateness of the interventional procedures. This is an important aspect of disparities that needs to be understood; however, this is likely on the causal pathway and, therefore, not a confounder of the estimates presented in this study.

\section{Conclusions}

In summary, over the past 18 years we observed declines in hospitalization rates and mortality and increases in revascularization for AMI in the US Medicare population. We found that disparities by sex and also by race in hospitalization rates, receipt of revascularization procedures and mortality have persisted over time. Our study provides evidence of the continued challenge of insuring equal care to all patient groups.

\section{Additional files} Additional file 1: Flow Chart showing patient selection for AMI
cohort for our study.

Additional file 2: Percentage of White and Black Men and Women who underwent Coronary Angiography within 30-day of admission for AMI.

Additional file 3: Percentage of White and Black Men and Women who underwent percutaneous coronary intervention $(\mathrm{PCI})$ within 30-day of admission for AMI.

Additional file 4: Percentage of White and Black Men and Women who underwent Coronary Artery Bypass Graft (CABG) within 30-day after admission for AMI.

Additional file 5: Procedural Utilization During the Index Admission for initial AMI Adjusted for Patient Age and Comorbidities.

Additional file 6: Procedural Utilization During the Index Admission for initial AMI Adjusted for Patient Age and Comorbidities, and Clustering of Patients within Hospitals.

\section{Competing interests}

There are no financial conflicts directly related to this study. Authors declare the following financial relationships with any organisations that might have an interest in the submitted work in the previous three years. JAS has received investigator-initiated research grants from Takeda and Savient; consultant fees from Takeda, Savient, Regeneron and Allergan; and is a member of the executive of OMERACT, an organization that develops outcome measures in rheumatology and receives arms-length funding from 36 companies. Dr. Singh is also a member of the American College of Rheumatology's Guidelines Subcommittee of the Quality of Care Committee and Veterans Affairs Rheumatology Field Advisory Committee. Dr. Cram,

Dr. Ibrahim and Xin Lu have no competing interests.

\section{Authors' contributions}

Study concept and design: JAS, XL and PC. Acquisition of data: $\mathrm{XL}$ and $\mathrm{SI}$. Analysis and interpretation of data: JAS, XL, SI and PC. Drafting of the manuscript: SI and PC. Critical revision of the manuscript for important intellectual content: JAS, XL, SI and PC. Statistical analysis: XL. Obtained funding: PC. Administrative, technical, or material support: SI and PC. Study supervision: SI and PC. PC had full access to all the data in the study and takes responsibility for the integrity of the data and the accuracy of the analysis. All statistical analyses were performed at the University of lowa by $\mathrm{XL}$ with supervision by PC. All authors read and approved the final manuscript.

\section{Acknowledgements}

This work is also funded in part by R01 HL085347 from NHLBI and R01 AG033035 from NIA at the NIH. JAS is supported by research grants from National Institute of Aging, National Cancer Institute, Agency for Health Quality and Research Center for Education and Research on Therapeutics (CERTS), and the resources and the use of facilities at the Birmingham VA Medical Center, Alabama, USA. PC is supported by a K24 award from NIAMS (AR062133) and by the Department of Veterans Affairs. Dr. Ibrahim is supported by Grant Number K24AR055259 from the National Institute of Arthritis and Musculoskeletal and Skin Diseases.

\section{Data sharing}

We will share data with any investigator interested in replicating these findings or interested in future collaborations, pursuant to institutional and IRB regulations, in accordance with patient privacy, confidentiality and HIPAA laws/regulations.

\section{Disclaimer}

The views expressed in this article are those of the authors and do not necessarily reflect the position or policy of the Department of Veterans Affairs or the United States government. 


\section{Author details}

'Medicine Service, Birmingham Veterans Affairs Medical Center, the University of Alabama at Birmingham, 510 S 20th Street, Faculty Office Tower 805B, Birmingham, AL 35294, UK. Division of General Internal Medicine, Department of Internal Medicine, University of lowa Carver College of Medicine and CADRE, lowa City Veterans Administration Medical Center, 451 Newton Road 200 Medicine Administration Building, lowa City, IA 52242, USA. ${ }^{3}$ Center for Health Equity Research and Promotion, Philadelphia Veterans Affairs Medical Center, the Perelman University of Pennsylvania School of Medicine, 3400 Spruce St, Philadelphia, PA 19104, USA. ${ }^{4}$ Division of General Internal Medicine and Geriatrics, University Health Network and Mount Sinai Hospitals, 200 Elizabeth Street, Eaton North 14th Floor, Toronto, ON M5G 2C4, Canada. ${ }^{5}$ Faculty of Medicine, University of Toronto, Toronto, ON, Canada.

Received: 21 June 2014 Accepted: 22 September 2014

Published online: 24 October 2014

\section{References}

1. Ani C, Pan D, Martins D, Ovbiagele B: Age- and sex-specific in-hospital mortality after myocardial infarction in routine clinical practice. Cardiol Res Pract 2010, 2010:752765.

2. Barnato AE, Lucas FL, Staiger D, Wennberg DE, Chandra A: Hospital-level racial disparities in acute myocardial infarction treatment and outcomes. Med Care 2005, 43:308-319.

3. Bertoni AG, Goonan KL, Bonds DE, Whitt MC, Goff DC Jr, Brancati FL: Racial and ethnic disparities in cardiac catheterization for acute myocardial infarction in the United States, 1995-2001. J Natl Med Assoc 2005, 97:317-323.

4. Bradley EH, Herrin J, Wang Y, McNamara RL, Webster TR, Magid DJ, Blaney M, Peterson ED, Canto JG, Pollack CV Jr, Krumholz HM: Racial and ethnic differences in time to acute reperfusion therapy for patients hospitalized with myocardial infarction. JAMA 2004, 292:1563-1572.

5. Bullen C, Beaglehole R: Ethnic differences in coronary heart disease case fatality rates in Auckland. Aust N Z J Public Health 1997, 21:688-693.

6. Institute of Medicine. 100 Initial Priority Topics for Comparative Effectiveness Research. National Academies Press; 2009. http://www.iom. edu/ /media/Files/Report\%20Files/2009/ComparativeEffectivenessResearch Priorities/Stand\%20Alone\%20List\%20of\%20100\%20CER\%20Priorities\%20-\% 20for\%20web.ashx.

7. European Commission. Public Health. The EU cohesion policy and health [http://ec.europa.eu/health/health_structural_funds/policy/index_en.htm]

8. World Conference on Social Determinants of Health [http://www.who.int/sdhconference/background/news/facts/en/]

9. Cartwright-Smith L, Rosenbaum S, Mehta D: Disparities Reduction and Minority Health Improvement under the ACA. Volume 3. Washington, D.C: The George Washington University School of Public Health and Health Services; 2011:1-6.

10. Jha AK, Fisher ES, Li Z, Orav EJ, Epstein AM: Racial trends in the use of major procedures among the elderly. N Engl J Med 2005, 353:683-691.

11. Escarce JJ, McGuire TG: Methods for using Medicare data to compare procedure rates among Asians, blacks, Hispanics, Native Americans, and whites. Health Serv Res 2003, 38:1303-1317.

12. Guadagnoli E, Landrum MB, Peterson EA, Gahart MT, Ryan TJ, McNeil BJ: Appropriateness of coronary angiography after myocardial infarction among Medicare beneficiaries. Managed care versus fee for service. N Engl J Med 2000, 343:1460-1466.

13. Peterson ED, Shaw LK, DeLong ER, Pryor DB, Califf RM, Mark DB: Racial variation in the use of coronary-revascularization procedures. Are the differences real? Do they matter? N Engl J Med 1997, 336:480-486.

14. Vaughan-Sarrazin MS, Hannan EL, Gormley CJ, Rosenthal GE: Mortality in Medicare beneficiaries following coronary artery bypass graft surgery in states with and without certificate of need regulation. JAMA 2002 288:1859-1866.

15. Elixhauser A, Steiner C, Harris DR, Coffey RM: Comorbidity measures for use with administrative data. Med Care 1998, 36:8-27.

16. Centers for Medicare and Medicaid Services: 2012 measures maintenance technical report: acute myocardial infarction, heart failure, and pneumonia 30-day risk-standardized mortality measures. http://www. henlearner.org/wp-content/uploads/2012/03/Hospital-Readmissions Metric-Maintenance-Technical-Report.pdf
17. Hardoon SL, Whincup PH, Petersen I, Capewell S, Morris RW: Trends in longer-term survival following an acute myocardial infarction and prescribing of evidenced-based medications in primary care in the UK from 1991: a longitudinal population-based study. J Epidemiol Community Health 2011, 65:770-774

18. Peterson ED, Shah BR, Parsons L, Pollack CV Jr, French WJ, Canto JG, Gibson CM, Rogers WJ: Trends in quality of care for patients with acute myocardial infarction in the National Registry of Myocardial Infarction from 1990 to 2006. Am Heart J 2008, 156:1045-1055.

19. Werner RM, Goldman LE, Dudley RA: Comparison of change in quality of care between safety-net and non-safety-net hospitals. JAMA 2008 , 299:2180-2187.

20. Chen J, Normand SL, Wang Y, Drye EE, Schreiner GC, Krumholz HM: Recent declines in hospitalizations for acute myocardial infarction for Medicare fee-for-service beneficiaries: progress and continuing challenges. Circulation 2010, 121:1322-1328.

21. Talbott EO, Rager JR, Brink LL, Benson SM, Bilonick RA, Wu WC, Han YY: Trends in acute myocardial infarction hospitalization rates for US States in the CDC tracking network. PLOS One 2013, 8:e64457.

22. Fang J, Alderman MH: Dissociation of hospitalization and mortality trends for myocardial infarction in the United States from 1988 to 1997. Am J Med 2002, 113:208-214.

23. Salomaa $V$, Koukkunen $H$, Ketonen $M$, Immonen-Raiha $P$, Karja-Koskenkari $P$, Mustonen J, Lehto S, Torppa J, Lehtonen A, Tuomilehto J, Kesäniemi YA Pyörälä K: A new definition for myocardial infarction: what difference does it make? Eur Heart J 2005, 26:1719-1725.

24. Schmidt M, Jacobsen JB, Lash TL, Botker HE, Sorensen HT: 25 year trends in first time hospitalisation for acute myocardial infarction, subsequent short and long term mortality, and the prognostic impact of sex and comorbidity: a Danish nationwide cohort study. BMJ 2012, 344:e356.

25. Go AS, Mozaffarian D, Roger VL, Benjamin EJ, Berry JD, Borden WB, Bravata DM, Dai S, Ford ES, Fox CS, Franco S, Fullerton HJ, Gillespie C, Hailpern SM, Heit JA, Howard VJ, Huffman MD, Judd SE, Kissela BM, Kittner SJ, Lackland DT, Lichtman JH, Lisabeth LD, Mackey RH, Magid DJ, Marcus GM, Marelli A, Matchar DB, McGuire DK, Mohler ER 3rd, et al: Heart disease and stroke statistics-2013 update: a report from the American Heart Association. Circulation 2013, 127:e6-e245.

26. Go AS, Mozaffarian D, Roger VL, Benjamin EJ, Berry JD, Blaha MJ, Dai S, Ford ES, Fox CS, Franco S, Fullerton HJ, Gillespie C, Hailpern SM, Heit JA, Howard VJ, Huffman MD, Judd SE, Kissela BM, Kittner SJ, Lackland DT, Lichtman JH, Lisabeth LD, Mackey RH, Magid DJ, Marcus GM, Marelli A, Matchar DB, McGuire DK, Mohler ER 3rd, Moy CS, et al: Heart disease and stroke statistics-2014 update: a report from the American Heart Association. Circulation 2014, 129:e28-e292.

27. Popescu I, Vaughan-Sarrazin MS, Rosenthal GE: Differences in mortality and use of revascularization in black and white patients with acute MI admitted to hospitals with and without revascularization services. JAMA 2007 297:2489-2495.

28. Brown CP, Ross L, Lopez I, Thornton A, Kiros GE: Disparities in the receipt of cardiac revascularization procedures between blacks and whites: an analysis of secular trends. Ethn Dis 2008, 18:S2-S112-S117.

29. Maynard C, Litwin PE, Martin JS, Cerqueira M, Kudenchuk PJ, Ho MT, Kennedy JW, Cobb LA, Schaeffer SM, Hallstrom AP, Weaver, WD: Characteristics of black patients admitted to coronary care units in metropolitan Seattle: results from the Myocardial Infarction Triage and Intervention Registry (MITI). Am J Cardiol 1991, 67:18-23.

30. Mukamel DB, Weimer DL, Mushlin Al: Referrals to high-quality cardiac surgeons: patients' race and characteristics of their physicians. Health Serv Res 2006, 41:1276-1295.

31. Vaccarino V, Rathore SS, Wenger NK, Frederick PD, Abramson IL, Barron HV, Manhapra A, Mallik S, Krumholz HM, National Registry of Myocardial Infarction I: Sex and racial differences in the management of acute myocardial infarction, 1994 through 2002. N Engl J Med 2005, 353:671-682.

32. Vaccarino V, Parsons L, Every NR, Barron HV, Krumholz HM: Sex-based differences in early mortality after myocardial infarction. National Registry of Myocardial Infarction 2 Participants. N Engl J Med 1999 341:217-225.

33. Iribarren C, Tolstykh I, Somkin CP, Ackerson LM, Brown TT, Scheffler R, Syme L, Kawachi I: Sex and racial/ethnic disparities in outcomes after acute myocardial infarction: a cohort study among members of a large 
integrated health care delivery system in northern California. Arch Intern Med 2005, 165:2105-2113.

34. Lehto HR, Lehto S, Havulinna AS, Ketonen M, Lehtonen A, Kesaniemi YA, Airaksinen K, Salomaa V: Sex differences in short- and long-term case-fatality of myocardial infarction. Eur J Epidemiol 2011, 26:851-861.

35. Vaidya D, Becker DM, Bittner V, Mathias RA, Ouyang P: Ageing, menopause, and ischaemic heart disease mortality in England, Wales, and the United States: modelling study of national mortality data. BMJ 2011, 343:d5170.

36. Nauta ST, Deckers JW, van Domburg RT, Akkerhuis KM: Sex-related trends in mortality in hospitalized men and women after myocardial infarction between 1985 and 2008: equal benefit for women and men. Circulation 2012, 126:2184-2189.

doi:10.1186/s12916-014-0190-6

Cite this article as: Singh et al:: Trends in and disparities for acute myocardial infarction: an analysis of Medicare claims data from 1992 to 2010. BMC Medicine 2014 12:190.

\section{Submit your next manuscript to BioMed Central and take full advantage of:}

- Convenient online submission

- Thorough peer review

- No space constraints or color figure charges

- Immediate publication on acceptance

- Inclusion in PubMed, CAS, Scopus and Google Scholar

- Research which is freely available for redistribution 